\title{
Toxic leukoencephalopathy
}

\section{Editorial}

Leukoencephalopathy means the disease of the cerebral white matter. Therefore, this disease can affect visual, motor and sensory systems. Additionally, Leukoencephalopathy can involve cognitive and emotional activities. The clinical manifestations of this disease depend on the affected part of the cerebral white matter and its intensity. The clinical manifestations include a spectrum ranging from inattention, forgetfulness and personality changes, which could be mistaken for psychiatric problems, to more severe cases such as muscle spasms, hemiparesis, dysarthria, ataxia, dementia, stupor, coma and death. ${ }^{1}$ There are different toxic factors causing toxic Leukoencephalopathy, for example, chronic use of ecstasy, heroin, cocaine, ethanol and methadone, as well as methadone or heroin overdoses. ${ }^{1-4}$

The diagnosis of toxic Leukoencephalopathy is based on clinical manifestations along with changes in brain white matter observed in the brain $\mathrm{T}_{2}$-weighted magnetic resonance imaging (MRI). ${ }^{1}$ Therefore, any neurological disorder, behavioral change, inattention, forgetfulness, and psychiatric disorder such as depression or anxiety observed in individuals using ecstasy, heroin, cocaine, ethanol and methadone, or any early or late abnormal psychiatric or neurological findings after recovery from heroin or methadone overdoses, can be due to toxic Leukoencephalopathy and for the diagnosis, a brain $\mathrm{T}_{2}$-weighted MRI from the patient should be provided. There is no treatment for toxic Leukoencephalopathy. In some cases, this disease is reversible and patients can be recovered spontaneously from toxic Leukoencephalopathy after some months. Some researchers recommend the administration of corticosteroids, antioxidants such as coenzyme Q (30mg QID), vitamin E, and vitamin C (each 2000mg QD) as a treatment for toxic Leukoencephalopathy. ${ }^{4}$
Volume 2 Issue 2 - 2016

\section{Hossein Sanaei-Zadeh}

Department of Forensic Medicine and Clinical Toxicology, Shiraz University of Medical Sciences, Iran

Correspondence: Hossein Sanaei-Zadeh, Shiraz University of Medical Sciences, Shiraz, Iran, Tel 7|439|8796,

Email sanaeizadeh@sums.ac.ir

Received: July 14, 2016 | Published: July 19, 2016

\section{Acknowledgements}

None.

\section{Conflict of interest}

The author declares no conflict of interest.

\section{References}

1. Filley CM, Kleinschmidt-DeMasters BK. Toxic leukoencephalopathy. $N$ Engl J Med. 2001;345(6):425-432.

2. Buxton JA, Sebastian R, Clearsky L, et al. Chasing the dragon characterizing cases of leukoencephalopathy associated with heroin inhalation in British Columbia. Harm Reduct. 2011;8:3.

3. Salgado RA, Jorens PG, Baar I, et al. Methadone-induced toxic leukoencephalopathy: MR imaging and MR proton spectroscopy findings. AJNR Am J Neuroradiol. 2010;31(3):565-566.

4. Kriegstein AR, Shungu DC, Millar WS, et al. Leukoencephalopathy and raised brain lactate from heroin vapor inhalation ("chasing the dragon"). Neurology. 1999;53(8):1765-1773. 